\title{
Application of Forward Modelling and Dictionary Indexing to EBSD Orientation Data as a Means of Quantifying Dislocation Substructure Formation in FCC Metals
}

Daniel L. Foley ${ }^{1}$, Cassandra Pate ${ }^{1}$, Kyle Matthews ${ }^{1}$, Xingyuan Zhao $^{2}$, Nicolas Savino ${ }^{2}$, Marc DeGraef $^{3}$, Leslie Lamberson $^{2}$, Mitra L. Taheri ${ }^{1 *}$

${ }^{1 .}$ Department of Materials Science and Engineering, Drexel University, Philadelphia. PA, USA.

2. Department of Mechanical Engineering and Mechanics, Drexel University, Philadelphia, PA, USA.

3. Department of Materials Science and Engineering, Carnegie Mellon University, Pittsburgh, PA, USA.

*Corresponding author: mlt48@drexel.edu

The strain hardening behavior of FCC metals is quite sensitive to strain rate when compared to BCC and HCP metals, and this is generally attributed to the development of different dislocation substructures at different strain rates [1]. At quasistatic (QS) strain rates these metals develop a cellular substructure via cross-slip in a process known as dynamic recovery. High rate deformation, on the other hand, suppresses dynamic recovery and leads to a planar substructure or a more diffuse dislocation tangle and enhanced strain hardening [2]. Sufficiently high strain rate leads to the nucleation of deformation twins, which further increase the strain hardening response [3]. Furthermore, an orientation dependence on substructure and twin formation has been observed whereby individual grains can be favorably oriented for either cellular or planar substructure and twin development [4]. This high strain hardenability observed under dynamic deformation conditions is desirable for many high-speed and structural applications, and therefore understanding the mechanism through which it arises is crucial. Though these dislocation substructures have long been observed, quantification of dislocation densities and substructure size is an active topic of research and is necessary to better understand the slip-twin transition.

In the present study, pure copper is subjected to both QS $\left(10^{-3} \mathrm{~s}^{-1}\right)$ and dynamic $\left(10^{3} \mathrm{~s}^{-1}\right)$ compression. Dynamic deformation was done via the Kolsky (split-Hopkinson) method in conjunction with digital image correlation (DIC) in order to correlate continuum and microstructural results. Microstructural characterization was done in both scanning (SEM) and transmission (TEM) electron microscopes. Orientation data was acquired via electron backscatter diffraction (EBSD) in the SEM and precession electron diffraction (PED) in the TEM. Utilizing a method developed by Leff et. al. [5], we used this orientation data to both quantify and spatially resolve geometrically necessary dislocation (GND) density, thereby allowing us to quantify dislocation substructures. This method estimates GND density based on the Nye tensor, which can be retrieved by measuring orientation gradients within a crystal. Each pixel in the orientation scan is then assigned a density value, thereby allowing spatial resolution of GND densities. This is supplemented by the use of a dictionary method for indexing orientation data based on forward modelling of EBSD scattering developed by Singh and DeGraef [6]. This method cross references experimental diffraction patterns with a dictionary of simulated patterns and assigns the data the orientation of the best match, which significantly limits noise.

In this study we investigate strain rate dependent microstructural evolution in terms of the development of cellular and planar dislocation substructures, as well as the suppression of dynamic recovery. We evaluate the ability of using Nye tensor-based GND quantification and mapping as a means to identify unique dislocation substructures that develop as a result of varying strain rate. By comparing the SEM and TEM data, we can judge whether we are accurately measuring these nanoscale structures at the mesoscale, which would link relevant length-scales and allow for more complete evaluation of microstructural evolution. Furthermore, we also assess the benefit of using the dictionary indexing method with respect 
to the estimation GND density and substructure morphology and size. Together, these findings lay a groundwork for comprehensive methodology for quantitative analysis of dislocation substructure evolution.

\section{References:}

[1] PS Follansbee, Metall. Appl. Shock. high strain rate Phenom. 1(1), p. 451.

[2] GT (Rusty) Gray, Annu. Rev. Mater. Res. 42(1) (2012), p. 285.

[3] A Rohatgi, KS Vecchio and GT Gray, Metall. Mater. Trans. A 32(1) (2001),p. 135.

[4] I Gutierrez-Urrutia and D Raabe, Mater. Sci. Forum 702-703 (2011), p. 523.

[5] AC Leff, CR Weinberger and ML Taheri, Ultramicroscopy 153 (2015), p. 9.

[6] S Singh and M De Graef, Model. Simul. Mater. Sci. Eng. 24(8) (2016).
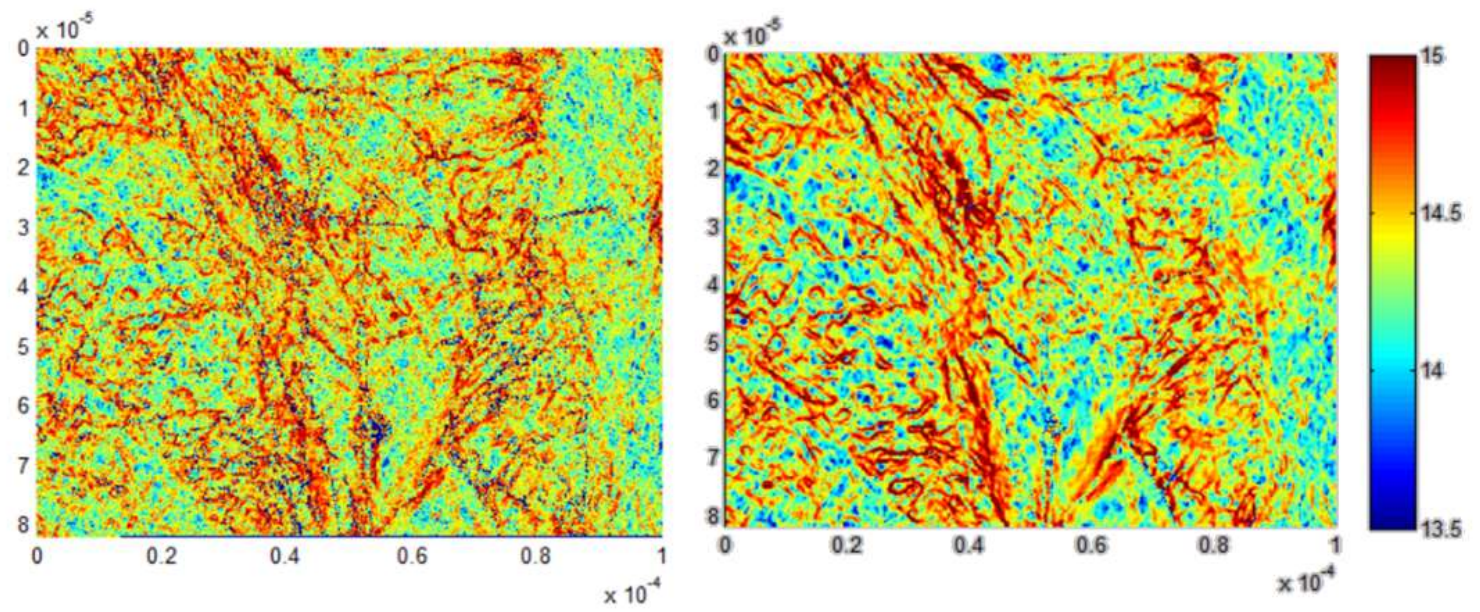

Figure 1. GND density maps for copper deformed at 3000s-1. Left, map based on raw EBSD data. Right, map based on dictionary-indexed EBSD data 